\title{
A novel adenovirus of Western lowland gorillas (Gorilla gorilla gorilla)
}

\author{
Diana Wevers ${ }^{1}$, Fabian H Leendertz ${ }^{2}$, Nelly Scuda ${ }^{1}$, Christophe Boesch³ ${ }^{3}$ Martha M Robbins ${ }^{3}$, Josephine Head ${ }^{3}$, \\ Carsten Ludwig ${ }^{4}$, Joachim Kühn ${ }^{5}$, Bernhard Ehlers ${ }^{1 *}$
}

\begin{abstract}
Adenoviruses (AdV) broadly infect vertebrate hosts including a variety of primates. We identified a novel AdV in the feces of captive gorillas by isolation in cell culture, electron microscopy and PCR. From the supernatants of infected cultures we amplified DNA polymerase (DPOL), preterminal protein (pTP) and hexon gene sequences with generic pan primate AdV PCR assays. The sequences in-between were amplified by long-distance PCRs of 2 - $10 \mathrm{~kb}$ length, resulting in a final sequence of $15.6 \mathrm{~kb}$. Phylogenetic analysis placed the novel gorilla AdV into a cluster of primate AdVs belonging to the species Human adenovirus B (HAdV-B). Depending on the analyzed gene, its position within the cluster was variable. To further elucidate its origin, feces samples of wild gorillas were analyzed. AdV hexon sequences were detected which are indicative for three distinct and novel gorilla HAdV-B viruses, among them a virus nearly identical to the novel AdV isolated from captive gorillas. This shows that the discovered virus is a member of a group of HAdV-B viruses that naturally infect gorillas. The mixed phylogenetic clusters of gorilla, chimpanzee, bonobo and human AdVs within the HAdV-B species indicate that host switches may have been a component of the evolution of human and non-human primate HAdV-B viruses.
\end{abstract}

\section{Findings}

Adenoviruses are non-enveloped icosahedral doublestranded DNA viruses that infect fish, amphibians, reptiles, birds and mammals [1]. Human adenoviruses (HAdV) are categorized into seven species (HAdV-A to HAdV-G) [2]. Each species includes a distinct number of serotypes [3]. In addition, intra-species shuffling of penton base, fiber and hexon genes by recombination has been frequently observed [4-6]. Simian adenoviruses have been discovered in monkeys and great apes [7-11]. They are very similar to $\mathrm{HAdV}$, and most of them can be grouped into corresponding HAdV species or the newly established species Simian adenovirus A (SAdV-A).

In 2008, a group of Western lowland gorillas (Gorilla gorilla gorilla) suffered from prolonged diarrhea and self-limiting respiratory disease in the Zoological gardens of Münster, Germany. To isolate viral agents potentially responsible for the symptoms, fecal samples were suspended in phosphate-buffered saline, sterile filtered and cultured on MRC-5 cells and A549 cells.

\footnotetext{
* Correspondence: ehlersb@rki.de

${ }^{1}$ FG12 Division of Viral Infections, Robert Koch Institute, Berlin, Germany Full list of author information is available at the end of the article
}

After eight days of culture, a cytopathogenic effect was observed. The culture supernatant was examined by electron microscopy, and virus-like structures were detected their size and general structure being consistent with that of adenoviruses (Additional Figure 1).

DNA was then extracted from culture supernatant using the Qiagen tissue kit according to the manufacturer's instructions (Qiagen, Hilden, Germany), and a generic primate adenovirus PCR was performed. For this purpose, a nested set of degenerate and deoxyinosine-substituted (deg/dI) primers was designed, targeting a highly conserved region of the DNA polymerase (DPOL) gene of primate mastadenoviruses (Figure 1; Table 1). PCR was performed in a total volume of $25 \mu \mathrm{l}$ with $0.2 \mu \mathrm{l}$ AmpliTaq Gold (Applied Biosystems), 20 pmol of each primer, $200 \mu \mathrm{M}$ dNTPs, $2 \mathrm{mM} \mathrm{MgCl}$, and 5\% DMSO. A T-Gradient thermocycler from Biometra was used with the following cycling conditions: $95^{\circ} \mathrm{C}$ for $12 \mathrm{~min}$, and $45 \mathrm{cycles}$ of $95^{\circ} \mathrm{C}$ for $30 \mathrm{sec}, 45^{\circ} \mathrm{C}$ $\left(1^{\text {st }}\right.$ round and $2^{\text {nd }}$ round) for $30 \mathrm{sec}$ and $72^{\circ} \mathrm{C}$ for $2 \mathrm{~min}$, followed by a $15 \mathrm{~min}$ final extension step at $72^{\circ} \mathrm{C}$. PCR products were purified using the Invisorb DNA clean up kit according to the instructions of the manufacturer (Invitek, Berlin, Germany), and directly 


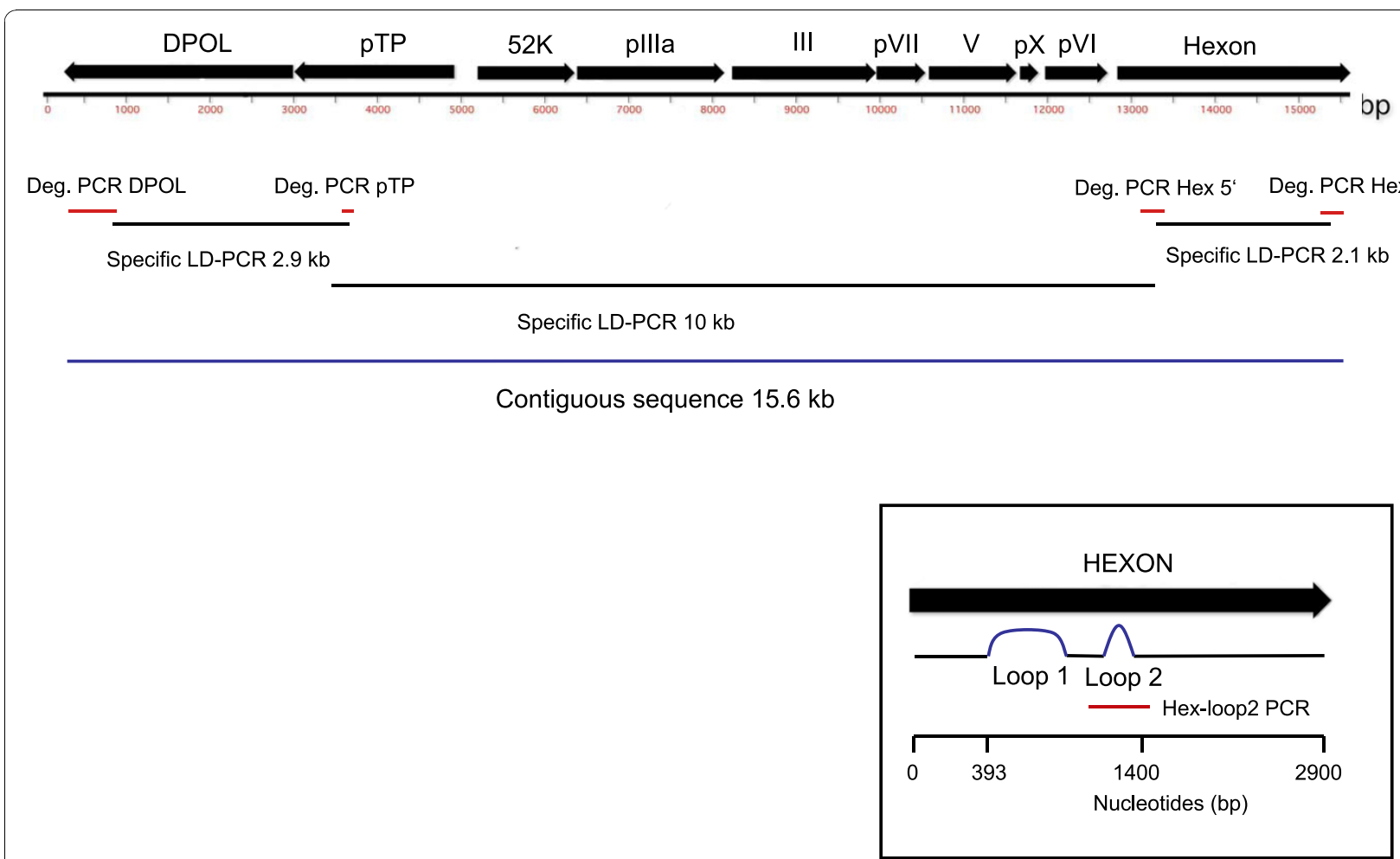

Figure 1 PCR amplification strategy. Above the ruler, the amplified part of the AdV genome is depicted. Below, a schematic visualisation of the positions of the degenerated consensus PCRs (red lines), the long-distance PCRs (black lines) and the resulting contiguous sequence (blue line) is given. In the box, the hexon gene is magnified, and the positions of the hexon loops and the Hex-loop2 PCR (red line) are illustrated.

sequenced with the Big Dye terminator cycle sequencing kit (Applied Biosystems, Warrington, UK) on a 377 automated DNA sequencer (Applied Biosystems). In BLAST analysis of GenBank, the sequence was most closely related to chimpanzee AdVs and human AdVs of the species HAdV-B. There was less similarity to the six gorilla HAdV-B viruses (SAdV-27.2; SAdV-28.2; SAdV41.1; SAdV-41.2; SAdV-46; SAdV-47; Table 2) recently described [12]. Since the novel gorilla AdV was the seventh HAdV-B of this host, it was named for the purpose of this paper Gorilla gorilla adenovirus B7 (GgorAdV-B7).

To acquire extended sequence information of GgorAdV-B7, three additional nested PCR assays were designed (Table 1) targeting the preterminal protein (pTP) and two conserved regions at the 5'- and 3'-end of the hexon gene (Figure 1). PCRs were performed as described above, except that elongation at $72^{\circ} \mathrm{C}$ was for $1 \mathrm{~min}$. With each primer set products of the expected size were obtained. BLAST analysis of their sequences also revealed a HAdV-B-like virus (not shown). To prove that the DPOL, PTP and hexon sequences originate from the same virus, we connected them with long-distance (LD) PCRs (Figure 1) using the TaKaRaEX PCR system according to the instructions of the manufacturer (Takara Bio Inc., Otsu, Japan). The LD primer pairs are listed with their annealing temperatures in Table 1. Three overlapping PCR products were generated and sequenced by primer walking. A final contiguous sequence of $15637 \mathrm{bp}$ was obtained spanning the genes DPOL, pTP and $52 \mathrm{k}$, the genes encoding the AdV proteins pIIIa, III (penton base), pVII, V, pX and $\mathrm{pVI}$, and the hexon gene of GgorAdV-B7 (Figure 1).

Since the AdV hexon gene is an important member of the core gene set used for AdV classification [2,3,13,14], we compared the available partial hexon gene of GgorAdV-B7 $(2.7 \mathrm{~kb})$ pair-wise with the corresponding hexon sequences of the most closely related human and chimpanzee HAdV-B viruses and all published gorilla HAdV-B viruses. The highest identity percentages were 96.6\% for SAdV-35.1 (chimpanzee AdV) and 96\% for HAdV-B21. The gorilla AdV SAdV-27.2, SAdV-28.2, SAdV-41.1, SAdV-41.2, SAdV-46 and SAdV-47 revealed only $87-91 \%$ identity. This closer relationship to HAdVB21 and SAdV-35.1 was restricted to the loop-encoding regions 1 and 2 [15] as visible in an analysis with the software SIMPLOT http://sray.med.som.jhmi.edu (Figure 2). In pair-wise comparisons of DPOL and pTP genes, GgorAdV-B7 was equally closely related to chimpanzee and gorilla HAdV-B viruses (96-99.9\%). 
Table 1 Primers for amplification of DPOL, pTP and hexon gene sequences

\begin{tabular}{|c|c|c|c|c|c|}
\hline Primer-set abbreviation & Targeted gene & Name of primer & sequence $5^{\prime}-3^{\prime}$ & PCR length & Annealing temp. \\
\hline \multicolumn{6}{|l|}{ Degenerate primers } \\
\hline \multirow[t]{4}{*}{ DPOL-cons } & DPOL & $4431-s^{\$}$ & GTnTwyGAyAThTGyGGhATGTAyGC & & \\
\hline & & 4428-as & GAGGCTGTCCGTrTC(n/i)CCGTA ${ }^{\#}$ & $956 \mathrm{bp}$ & $45^{\circ} \mathrm{C}$ \\
\hline & & $4428-5$ & CGGACGCCTCTGyTGGAC(n/i)AA & & \\
\hline & & 4429-as & GGCCAGCACrAA(n/i)GArGC & $650 \mathrm{bp}$ & $45^{\circ} \mathrm{C}$ \\
\hline \multirow[t]{4}{*}{ Hex-5'-cons } & Hexon & $4515-s$ & GTGGATGG(n/i)GA(r/i)GG(n/i)TACA & & \\
\hline & & 4515-as & CGCACAACGTC(r/i)AA(n/i)AC(y/i)TC & $536 \mathrm{bp}$ & $45^{\circ} \mathrm{C}$ \\
\hline & & $4516-5$ & TGTAACATGAC(y/i)AA(r(i)GA(y/i)TGG & & \\
\hline & & 4516-as & CAGGGCCCCCAT(n/i)GACA & $381 \mathrm{bp}$ & $45^{\circ} \mathrm{C}$ \\
\hline \multirow[t]{4}{*}{ Hex3'-cons } & Hexon & $4517-s$ & CGCAATGGTC(n/i)TACATGCAC & & \\
\hline & & 4517-as & CAGTGCCCGA(r/i)TA(k/i)GG(n/i)TT & $340 \mathrm{bp}$ & $45^{\circ} \mathrm{C}$ \\
\hline & & $4518-5$ & GCAGGACGC(y/i)TCGGAGTA & & \\
\hline & & 4518-as & $\operatorname{CACCC}(k / i) G T(r / i) T C(n / i) C C$ & $230 \mathrm{bp}$ & $45^{\circ} \mathrm{C}$ \\
\hline \multirow[t]{4}{*}{ pTP-cons } & pTP & $4521-s$ & TGGCGACGT(n/i)GT(n/i)TACAG & & \\
\hline & & 4521-as & CGGACT(y/i)(K/i)GA(r/i)CCTGAAA & $260 \mathrm{bp}$ & $45^{\circ} \mathrm{C}$ \\
\hline & & $4522-s$ & TACAGCCG(n/i)GTSTGGAAC & & \\
\hline & & 4522-as & CTGAAAGAGAGTTC(n/i)ACAGAATCA & $230 \mathrm{bp}$ & $45^{\circ} \mathrm{C}$ \\
\hline \multicolumn{6}{|c|}{ Specific primers for long-distance PCR } \\
\hline \multirow[t]{2}{*}{ pTP-DPOL-LD } & pTP, DPOL & 4659-s & TCGCATCTCCAACGACCT & & \\
\hline & & 4659-as & GCATCCATGGTGAAGATTCC & $2350 \mathrm{bp}$ & $60^{\circ} \mathrm{C}$ \\
\hline \multirow[t]{2}{*}{ Hex-LD } & Hexon & $4618-5$ & AGTTCGCTACACACTGGCTG & & \\
\hline & & 4618-as & ATTGCGGTGATGATTGAATG & 1354 bp & $59^{\circ} \mathrm{C}$ \\
\hline \multirow[t]{2}{*}{ pTP-Hex-LD } & pTP, Hexon & $4662-s$ & CTCGGTATCGTTGACGGC & & \\
\hline & & 4662-as & GATCAACGGGCACAAAGC & $10044 \mathrm{bp}$ & $60^{\circ} \mathrm{C}$ \\
\hline \multicolumn{6}{|c|}{ Primers specific for AdV species B } \\
\hline \multirow[t]{4}{*}{ Hex-loop2 } & Hexon & $5442 s$ & GAACAAGATACTITAGCATGTGGAA & & \\
\hline & & 5442 as & GATTGAATGGATTAACATTGTCC & $468 \mathrm{bp}$ & $55^{\circ} \mathrm{C}$ \\
\hline & Hexon & $5443 \mathrm{~s}$ & TAGAAAATCACGGGGTGGAAGA & & \\
\hline & & 5443as & GGCATCCAAAGACCATCTG & $380 \mathrm{bp}$ & $55^{\circ} \mathrm{C}$ \\
\hline
\end{tabular}

\$ $=$ sense, as $=$ antisense ${ }^{\#} \mathrm{I}=$ inosine

However, the penton base gene of GgorAdV-B7 showed a striking similarity (99.7\%) only to that of SAdV-29 (chimpanzee AdV). Using the program mVISTA http:// genome.lbl.gov/vista/index.shtml, the near-perfect match of the GgorAdV-B7 and SAdV-29 penton base genes over the entire gene length is clearly visible (Figure 3).

With the PhyML plug-in 2.0.1 of the Geneious Pro 5.0.4 software, phylogenetic trees were constructed on the basis of hexon, DPOL, pTP and penton base gene alignments (Figures 4). All published, completely sequenced $H A d V-B$ viruses were included. In the hexon-based tree, GgorAdV-B7 clustered with HAdVB21, SAdV-35.1 (chimpanzee AdV) and SAdV-35.2 (bonobo AdV) (Figure 4a). DPOL and pTP analyses placed GgorAdV-B7 into a tight cluster of gorilla and chimpanzee AdVs (Figure $4 \mathrm{~b}$ and $4 \mathrm{c}$ ). In the tree derived from the penton base, GgorAdV-B7 branched separately, nearly at the same position as SAdV-29
(Figure 4d). With the MrBayes 2.0.2 plug-in (Geneious Pro) or the Neighbor-Joining module of MacVector 10.6, trees with the same AdV clusters and similarly supported topology were obtained (data not shown).

The remarkably close relatedness of GgorAdV-B7 to chimpanzee AdVs (SAdV-29 and 35.1) prompted us to investigate whether gorillas naturally host GgorAdV-B7. For this purpose, we examined wild Western lowland gorillas (Gorilla. g. gorilla) from Gabon and additional captive gorillas. Fecal samples were collected from 19 individuals in a remote area with little human presence in Loango National Park, Gabon. They originated from fresh nest sites or were freshly found on gorilla paths [16]. Samples were collected using single-use gloves and preserved by drying over silica. DNA was extracted following a previously described method [17]. In addition, ten necropsy samples (spleen, liver, pancreas, lymph node, tonsil, lung, kidney, urine) and one plasma sample 
Table 2 Adenoviruses, accession numbers and hosts

\begin{tabular}{|c|c|c|c|c|c|}
\hline Adenovirus & Abbreviation & GenBank accession number & Host & Wild (Gabon) & Captive \\
\hline \multicolumn{6}{|l|}{ HAdV-B of this study } \\
\hline Gorilla gorilla adenovirus B7 & GgorAdV-B7 & HQ292614 & Western lowland gorilla & + & + \\
\hline Gorilla gorilla adenovirus B8 & GgorAdV-B8 & HQ292615 & Western lowland gorilla & + & + \\
\hline Gorilla gorilla adenovirus B9 & GgorAdV-B9 & HQ292616 & Western lowland gorilla & & + \\
\hline Gorilla gorilla adenovirus B10 & GgorAdV-B10 & HQ292617 & Western lowland gorilla & + & \\
\hline \multicolumn{6}{|l|}{ Published HAdV-B } \\
\hline Simian adenovirus 21 & SAdV-21 & AC000010 & Chimpanzee & & + \\
\hline Simian adenovirus 27.1 & SAdV-27.1 & FJ025909 & Chimpanzee & & + \\
\hline Simian adenovirus 27.2 & SAdV-27.2 & FJ025928 & Gorilla & & + \\
\hline Simian adenovirus 28.1 & SAdV-28.1 & FJ025914 & Chimpanzee & & + \\
\hline Simian adenovirus 28.2 & SAdV-28.2 & FJ025915 & Gorilla & & + \\
\hline Simian adenovirus 29 & SAdV-29 & FJ025916 & Chimpanzee & & + \\
\hline Simian adenovirus 32 & SAdV-32 & FJ025911 & Chimpanzee & & + \\
\hline Simian adenovirus 33 & SAdV-33 & JF025908 & Chimpanzee & & + \\
\hline Simian adenovirus 35.1 & SAdV-35.1 & FJ025912 & Chimpanzee & & + \\
\hline Simian adenovirus 35.2 & SAdV-35.2 & FJ025910 & Bonobo & & + \\
\hline Simian adenovirus 41.1 & SAdV-41.1 & FJ025913 & Gorilla & & + \\
\hline Simian adenovirus 41.2 & SAdV-41.2 & FJ025927 & Gorilla & & + \\
\hline Simian adenovirus 46 & SAdV-46 & FJ025930 & Gorilla & & + \\
\hline Simian adenovirus 47 & SAdV-47 & FJ025929 & Gorilla & & + \\
\hline Human adenovirus B3 & HAdV-B3 & DQ086466 & Human & & \\
\hline Human adenovirus B7 & HAdV-B7 & AC000018 & Human & & \\
\hline Human adenovirus B11 & HAdV-B11 & AY163756 & Human & & \\
\hline Human adenovirus B14 & HAdV-B14 & AY803294 & Human & & \\
\hline Human adenovirus B16 & HAdV-B16 & AY601636 & Human & & \\
\hline Human adenovirus B21 & HAdV-B21 & AY601633 & Human & & \\
\hline Human adenovirus B34 & HAdV-B34 & AY737797 & Human & & \\
\hline Human adenovirus B35 & HAdV-B35 & AY271307 & Human & & \\
\hline Human adenovirus B50 & HAdV-B50 & AY737798 & Human & & \\
\hline
\end{tabular}

were collected from four deceased captive gorillas in the Zoological gardens of Berlin as well as six fecal samples from three captive gorillas in the Zoological gardens of Münster, Germany. To test for the presence of HAdV-B viruses, we set up a nested PCR (PCR Hex-loop2; Table 1) which targets flanking sequences of a hyper variable region (loop 2) in the hexon gene (Figure 1) and amplifies $380 \mathrm{bp}$. The primers were deduced from HAdV-B sequences only and not degenerated. A total of 36 gorilla samples were screened. AdV DNA was only detected in feces (5/19 wild gorilla samples and 4/6 captive gorilla samples). In total, 9/25 fecal samples were PCRpositive (36\%), and the products sequenced. Most importantly, a virus apparently identical to GgorAdVB7 was identified in a wild gorilla from Gabon. Three additional $\mathrm{HAdV}$-B viruses were also detected. Two were without close similarities to any published AdV sequence. The third one was nearly identical to the gorilla AdVs SAdV-27.2 and SAdV-47, which had been originally isolated from captive individuals [12]. They were tentatively named GgorAdV-B8, -B9 and -B10.

GgorAdV-B8 was detected in two fecal samples from wild gorillas in Gabon and in one sample from Münster. Its hexon sequence revealed the highest percentage of identity (86.5\%) to SAdV-21 (chimpanzee HAdV-B). GgorAdV-B9 was only amplified from captive gorillas (two fecal samples from Münster) and showed 88\% identity to SAdV-29 (chimpanzee HAdV-B). The GgorAdVB7 to -B10 Hex-loop2 sequences and closely related sequences of published gorilla, chimpanzee, bonobo and human HAdV-B viruses were subjected to phylogenetic analysis as described above. In the tree, the HAdV-B viruses segregate into several subclades with members of two, three or four host species (human, chimpanzee, bonobo and gorilla) (Figure 5). This mixed clustering was also observed upon analysis of the nearly complete hexon gene (2.7 kb; Figure 4a) with GgorAdV-B7 only. It was partially visible in the penton base tree (Figure $4 \mathrm{~d}$ ) and 


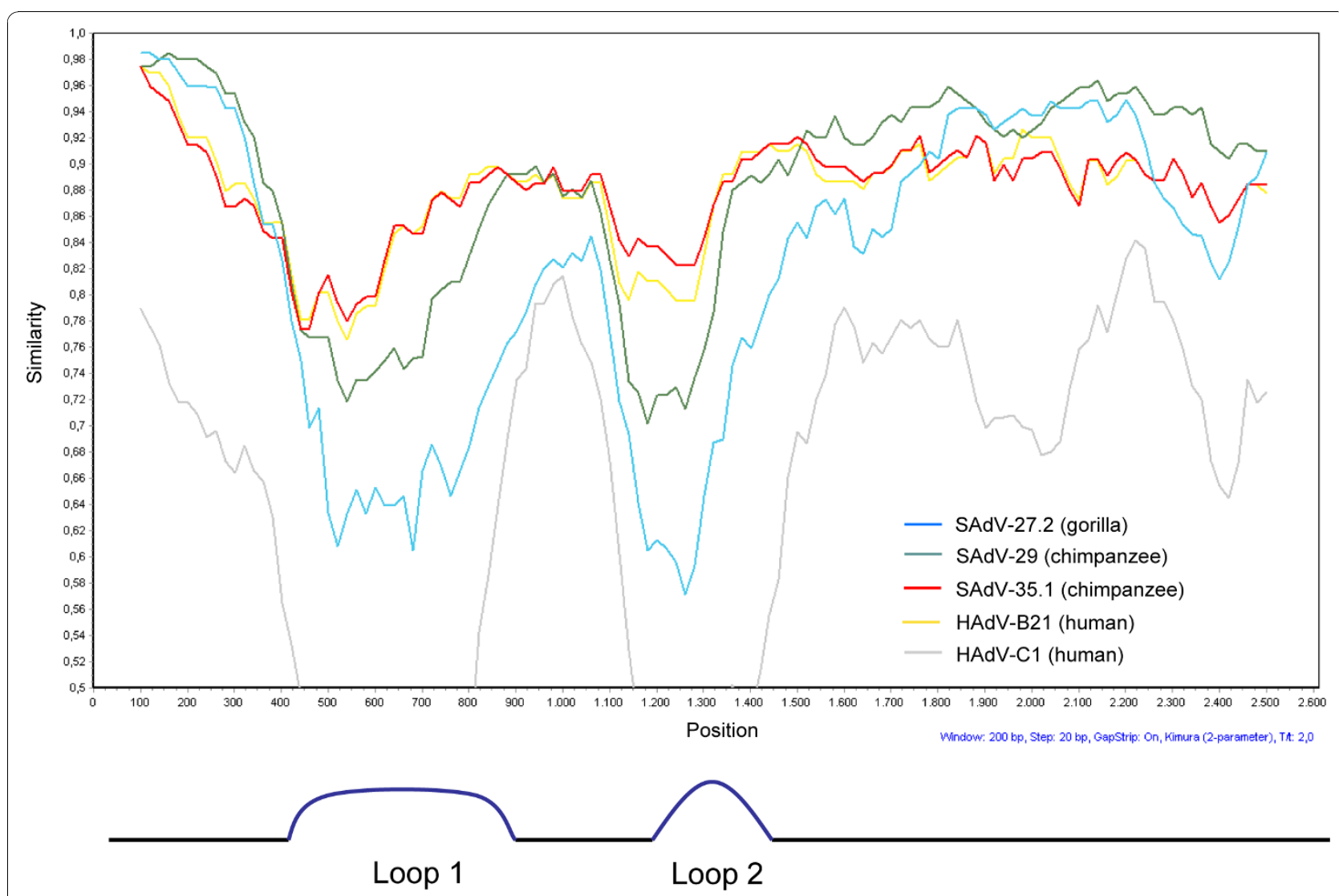

Figure 2 Simplot analysis of the hexon gene. $2.8 \mathrm{~kb}$ of the GgorAdV-B7 hexon gene were compared to the hexon genes of selected chimpanzee (green and red line), gorilla (blue line) and human (yellow and grey line) AdVs. Below the plot, the analysis parameters are listed in blue font. The hexon Loop 1 and Loop 2 regions are indicated at the bottom.

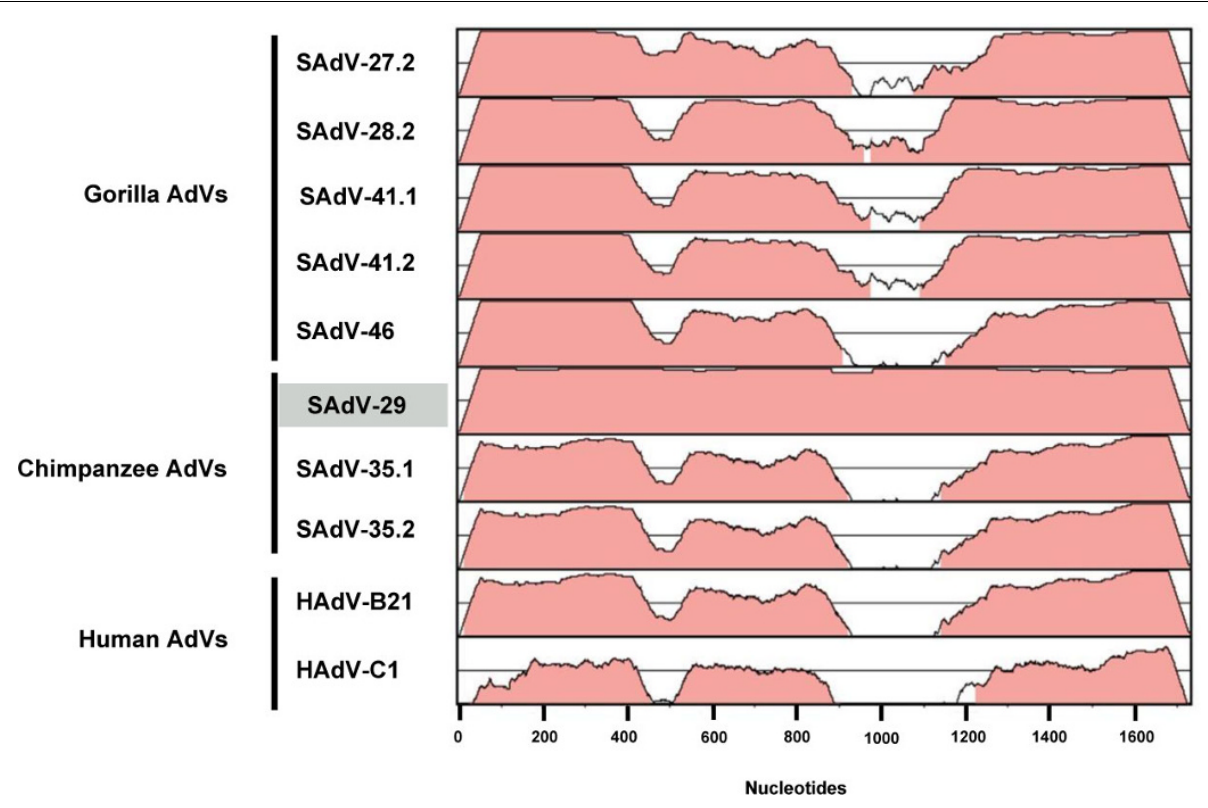

Figure 3 Pairwise sequence alignment of the penton base open reading frame. The penton base gene of GgorAdV-B7 was compared with those of five gorilla-, three chimpanzee- and two human AdVs. The 50-100 percent sequence conservation is represented by the height of each data point along the $y$ axis. The chimpanzee AdV SAdV-29 is highlighted in grey designating the exceptionally high similarity to GgorAdV-B7. 


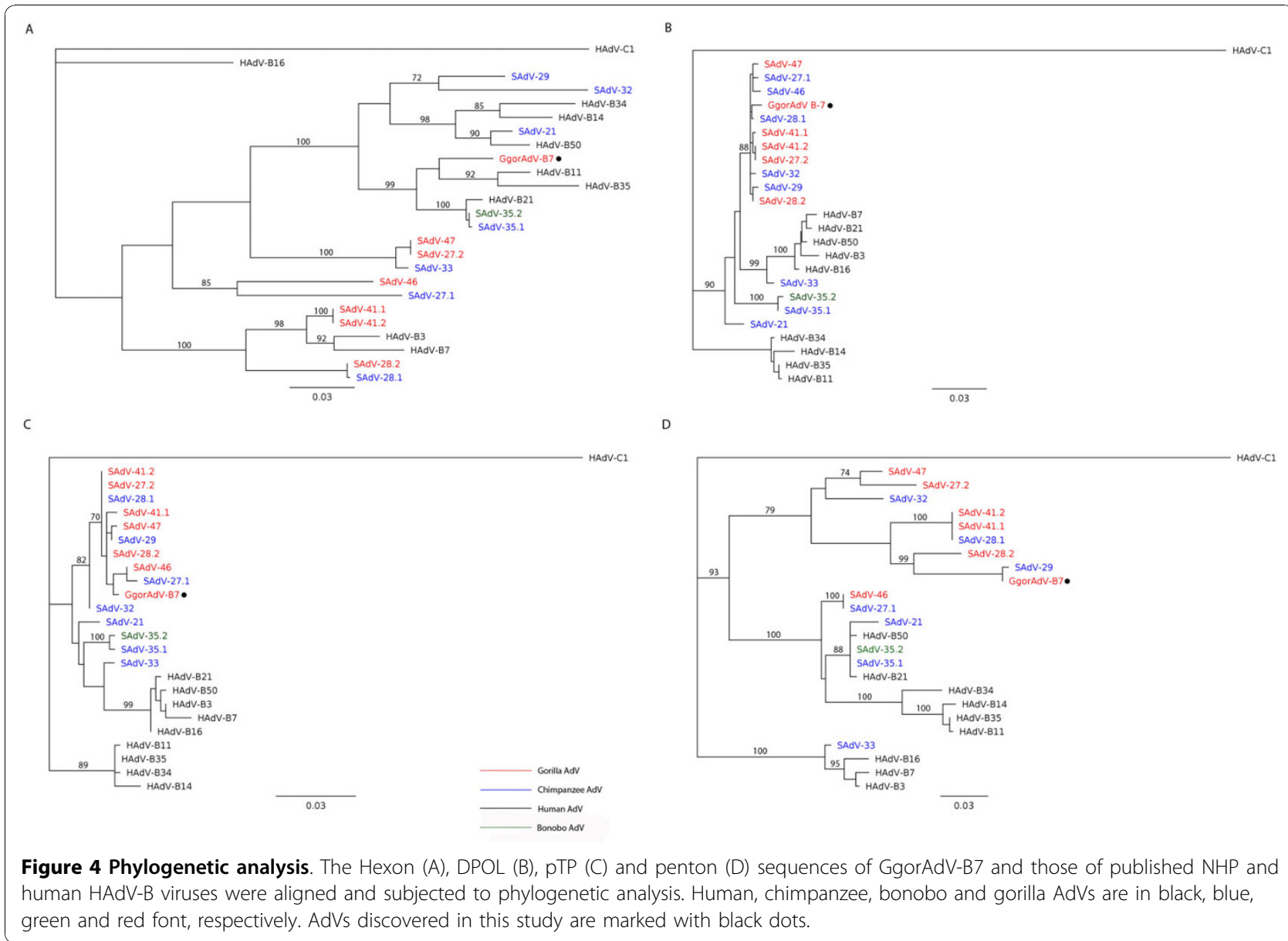

entirely absent from the DPOL and pTP trees (Figures $4 \mathrm{~b}$ and $4 \mathrm{c}$ ). In addition, the phylogenetic position of a given primate AdV frequently differed, depending on the analyzed gene (compare Figures 4a to 4d).

Taken together, these observations cannot be explained by co-speciation. Rather, they are in line with recombination and host switching. Such events have been previously discussed to be involved in the evolution of human AdV, because intra-species shuffling of penton base, fiber and hexon genes has been frequently observed [4-6]. In addition, a recombinant between viruses of the sub-clades HAdV-B1 and HAdV-B2 has been isolated from a captive chimpanzee [12]. Here, a close similarity of GgorAdV-B7 to SAdV-29 (complete sequence, excluding hexon loops) and to SAdV-35.1 (hexon loops) was observed (Figure 2). However, since in the loop region the nucleic acid identity between GgorAdV-B7 and SAdV-35.1 was well below 100\%, it is unlikely that the existing AdVs are parent viruses in a recent recombination event giving rise to GgorAdV-B7. Rather, a more ancient one with subsequent genetic drift may have been involved or recombination with an unknown AdV, as suggested for HAdV-A18 [18].
Shuffling of genes by recombination between AdVs that naturally infect different host species (e.g., great ape and human AdVs) but under certain conditions coinfect the same host, may be an additional mechanism by which AdVs exchange genetic information. This could occur in places where contacts between humans and apes are frequent like in zoos and animal facilities. In addition, people who are involved in hunting primates and preparation of bush meat [19] are at risk to be infected. So far, infections of humans with nonhuman primate (NHP) AdVs have not been observed. Nevertheless, antibodies with specificity for chimpanzee HAdV-C viruses have been detected in humans from Sub-Saharan Africa and were significantly less frequent in people from the United States of America and Thailand [20]. In addition, the species HAdV-E comprises only one human serotype but more than 12 great ape serotypes. Therefore, the human HAdV-E was thought to be the result of a zoonotic transmission from chimpanzees to humans [21]. The gorilla AdV described in the present study (GgorAdV-B7) is highly similar to chimpanzee AdVs. Thus, a transmission event between chimpanzees and gorillas was possibly involved which is 


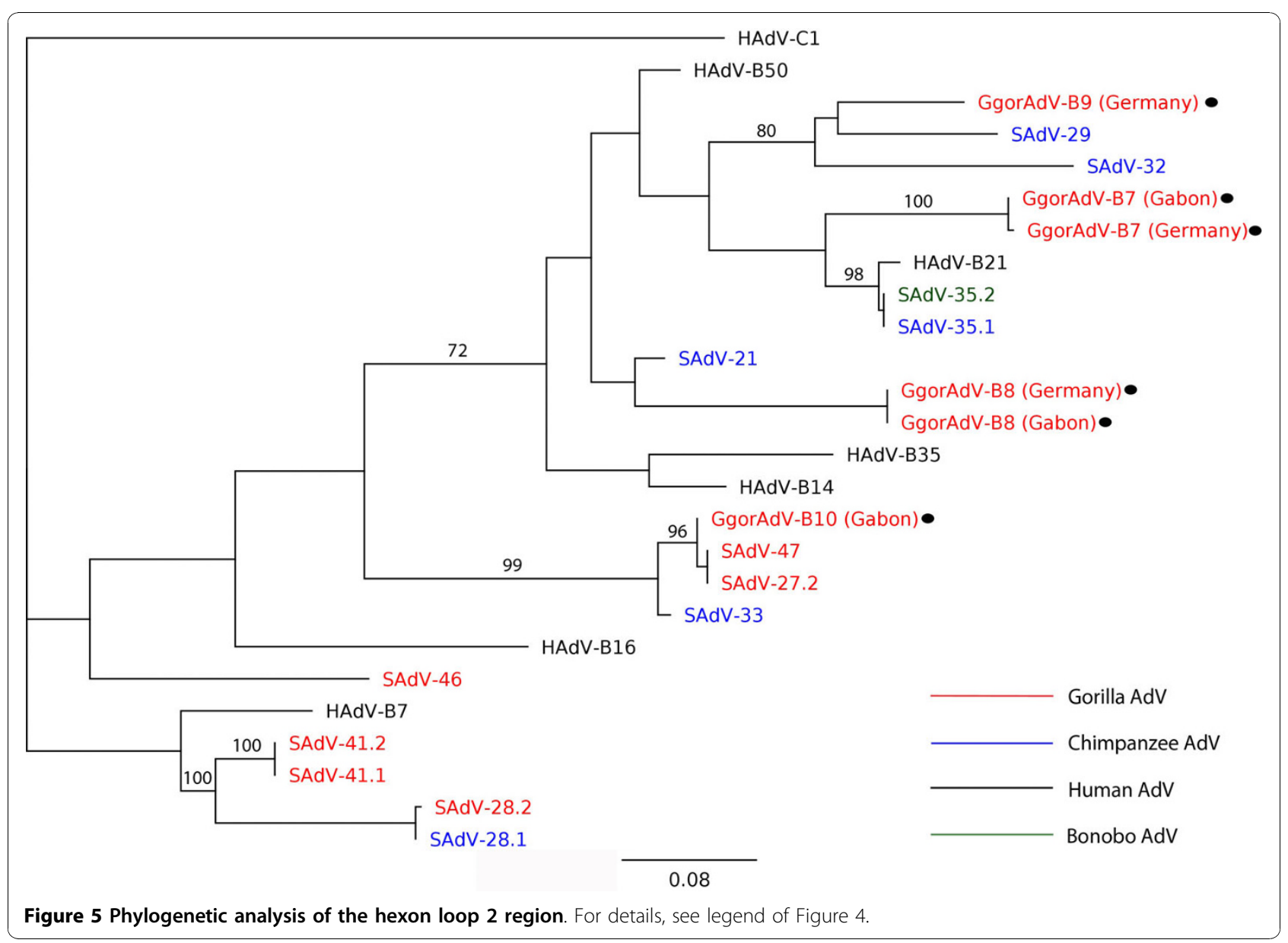

further indication for the potential of AdVs to jump between closely related hosts.

Very little is known about the pathogenic properties of NHP-AdV. GgorAdV-B7 was originally discovered in a group of gorillas suffering from prolonged diarrhea and self-limiting respiratory infection. Since human species B AdV have been linked to respiratory diseases [22-24], an etiological association of GgorAdV-B7 with the observed respiratory symptoms is possible. However, recent studies reported the frequent shedding of AdVs in the feces of healthy captive chimpanzees and gorillas $[12,25]$. Therefore, further investigations are needed.

Knowledge about the spectrum of AdV in wild great apes in general [25] is very limited. Specifically from wild gorillas, no information has been published. Although examining only a small set of samples, our findings show that AdV infecting captive gorillas can readily be found in wild animals (GgorAdV-B7; GgorAdV-B8). This is a good example of how humans may be brought into contact with new pathogens, not only locally through bushmeat hunting in regions where NHP live naturally, but also in other regions of the world where NHP are housed in zoos.
The high variety of known and novel HAdV-B viruses in great apes calls for larger studies to understand the diversity of AdVs currently circulating in African NHP as well as in local human populations. It is justified to assume that such studies will improve our insight into the zoonotic potential of adenoviruses and possibly answer the intriguing question whether AdVs of non-human primates have already contributed to the human "adeno-virosphere".

\section{Accession numbers}

The sequences reported in this study were deposited in GenBank under the accession numbers listed in Table 2.

\section{Additional material}

Additional Figure 1: Negative stain electron micrograph of adenovirus-like particles isolated from a fecal sample of a captive gorilla. Negatively stained with $1 \%$ uranyl acetate. Virus particles are $70-$ $90 \mathrm{~nm}$ in diameter with an icosahedral shape. Scale bar $=200 \mathrm{~nm}$.

\section{Acknowledgements}

We thank Michael Laue for electron microscopic analysis. The technical assistance of Sonja Liebmann and Cornelia Walter is kindly acknowledged. 
We thank the Agence Nationale des Parcs Nationaux (ANPN) and the Centre National de la Recherche Scientifique et Technique (CENAREST) of Gabon for permission to conduct research in Loango National Park. We also thank the Société pour la Conservation et le Développement (SCD) and Wildlife Conservation Society (WCS) for financial and logistical support. For sample collection from wild gorilla, we thank L. Rabanal, L. Mackaga, E. R. Guizard, N. Tagg, B. Graw, E. Fairet, M. Gregoire, L. Rankin and the other field assistants of the Loango Ape project. For DNA extraction and identification of individual samples from wild gorilla we thank M. Arandjelovic and L. Vigilant from the Max-Planck-Institute for Evolutionary Anthropology, Leipzig.

\section{Author details}

'FG12 Division of Viral Infections, Robert Koch Institute, Berlin, Germany. 2Junior research group "Emerging Zoonoses", Robert Koch Institute, Berlin, Germany. ${ }^{3}$ Department of Primatology, Max-Planck-Institute for Evolutionary Anthropology, Leipzig, Germany. ${ }^{4}$ Westfälischer Zoologischer Garten Münster $\mathrm{GmbH}$, Münster, Germany. Institute of Medical Microbiology, Clinical Virology, University of Münster, Münster, Germany.

\section{Authors' contributions}

DW and JK performed the cell culture experiments. DW, NS, JK and JH performed the molecular genetic studies. FHL, CB and MMR coordinated field work and sample collection. JH and CL sampled gorilla feces. DW, NS, $\mathrm{JK}, \mathrm{FHL}$ and $\mathrm{BE}$ conceived of the study, and participated in its design and coordination. DW, FHL, CB, JK and BE drafted the manuscript. All authors read and approved the final manuscript.

\section{Competing interests}

The authors declare that they have no competing interests.

Received: 1 September 2010 Accepted: 5 November 2010

Published: 5 November 2010

\section{References}

1. Davison AJ, Benko M, Harrach B: Genetic content and evolution of adenoviruses. J Gen Virol 2003, 84:2895-2908.

2. Benkö M, Harrach B, Both G, Russel W, Adair BM, Adam E, de Jong JC, Hess M, Johnson M, Kajon A, et al: Family Adenoviridae. In Virus Taxonomy: VIIIth Report of the International Committee on Taxonomy of Viruses. Edited by: Fauquet CM, Mayo MA, Maniloff J, Desselberger U, Ball LA. Elsevier; 2005:213-228.

3. Bailey A, Mautner V: Phylogenetic relationships among adenovirus serotypes. Virology 1994, 205:438-452.

4. Lukashev AN, Ivanova OE, Eremeeva TP, Iggo RD: Evidence of frequent recombination among human adenoviruses. Journal of General Virology 2008, 89:380-388.

5. Robinson CM, Rajaiya J, Walsh MP, Seto D, Dyer DW, Jones MS, Chodosh J: Computational analysis of human adenovirus type 22 provides evidence for recombination among species $\mathrm{D}$ human adenoviruses in the penton base gene. Journal of Virology 2009, 83:8980-8985.

6. Walsh MP, Chintakuntlawar A, Robinson CM, Madisch I, Harrach B, Hudson NR, Schnurr D, Heim A, Chodosh J, Seto D, Jones MS: Evidence of molecular evolution driven by recombination events influencing tropism in a novel human adenovirus that causes epidemic keratoconjunctivitis. PLoS One 2009, 4.

7. Kovacs G, Harrach B, Zakhartchouk AN, Davison AJ: Complete genome sequence of simian adenovirus 1: An Old World monkey adenovirus with two fiber genes. Journal of General Virology 2005, 86:1681-1686.

8. Basnight M Jr, Rogers NG, Gibbs CJ Jr, Gajdusek DC: Characterization of four new adenovirus serotypes isolated from chimpanzee tissue explants. American Journal of Epidemiology 1971, 94:166-171.

9. Kovacs GM, Davison AJ, Zakhartchouk AN, Harrach B: Analysis of the first complete genome sequence of an Old World monkey adenovirus reveals a lineage distinct from the six human adenovirus species. Journal of General Virology 2004, 85:2799-2807.

10. Bányai K, Esona MD, Liu A, Wang Y, Tu X, Jiang B: Molecular detection of novel adenoviruses in fecal specimens of captive monkeys with diarrhea in China. Veterinary Microbiology 2010, 142:416-419.

11. Kidd AH, Garwicz D, Oberg M: Human and simian adenoviruses: Phylogenetic inferences from analysis of VA RNA genes. Virology 1995, 207:32-45.
12. Roy S, Vandenberghe LH, Kryazhimskiy S, Grant R, Calcedo R, Yuan X, Keough M, Sandhu A, Wang Q, Medina-Jaszek CA, et al: Isolation and characterization of adenoviruses persistently shed from the gastrointestinal tract of non-human primates. PLOS Pathogens 2009, 5.

13. Casas I, Avellon A, Mosquera M, Jabado O, Echevarria JE, Campos RH, Rewers M, Perez-Brena P, Lipkin WI, Palacios G: Molecular identification of adenoviruses in clinical samples by analyzing a partial hexon genomic region. J Clin Microbiol 2005, 43:6176-6182.

14. Crawford-Miksza LK, Schnurr DP: Adenovirus serotype evolution is driven by illegitimate recombination in the hypervariable regions of the hexon protein. Virology 1996, 224:357-367.

15. Torres S, Chodosh J, Seto D, Jones MS: The revolution in viral genomics as exemplified by the bioinformatic analysis of human adenoviruses. Viruses 2010, 2:1367-1381.

16. Arandjelovic M, Head J, Kühl H, Boesch C, Robbins MM, Maisels F, Vigilant L: Effective non-invasive genetic monitoring of multiple wild western gorilla groups. Biological Conservation 2010, 143:1780-1791.

17. Nsubuga AM, Robbins MM, Roeder AD, Morin PA, Boesch C, Vigilant L: Factors affecting the amount of genomic DNA extracted from ape faeces and the identification of an improved sample storage method. Mol Ecol 2004, 13:2089-2094.

18. Walsh MP, Seto J, Tirado D, Chodosh J, Schnurr D, Seto D, Jones MS: Computational analysis of human adenovirus serotype 18. Virology 2010, 404:284-292.

19. Bennett EL, Blencowe E, Brandon K, Brown D, Burn RW, Cowlishaw G, Davies G, Dublin H, Fa JE, Milner-Gulland EJ, et al: Hunting for consensus: Reconciling bushmeat harvest, conservation, and development policy in West and Central Africa. Conservation Biology 2007, 21:884-887.

20. Xiang Z, Li Y, Cun A, Yang W, Ellenberg S, Switzer WM, Kalish ML, Ertl HCJ: Chimpanzee adenovirus antibodies in humans, sub-Saharan Africa. Emerging Infectious Diseases 2006, 12:1596-1599.

21. Purkayastha A, Ditty SE, SU J, McGraw J, Hadfield TL, Tibbetts C, Seto D: Genomic and bioinformatics analysis of HAdV-4, a human adenovirus causing acute respiratory disease: Implications for gene therapy and vaccine vector development. Journal of Virology 2005, 79:2559-2572.

22. Schmitz $\mathrm{H}$, Wigand $\mathrm{R}$, Heinrich W: Worldwide epidemiology of human adenovirus infections. American Journal of Epidemiology 1983, 117:455-466.

23. Russell WC: Adenoviruses. In Topley and Wilson's Virology Edited by: Mahy BWJ, Ter Meulen V, Hodder Arnold, 10 2005, 439-443.

24. Gray GC, Callahan JD, Hawksworth AW, Fisher CA, Gaydos JC: Respiratory diseases among U.S. Military personnel: Countering emerging threats. Emerging Infectious Diseases 1999, 5:379-387.

25. Tong S, Singh J, Ruone S, Humphrey C, Yip CCY, Lau SKP, Anderson LJ, Kaur T: Short report: Identification of adenoviruses in fecal specimens from wild chimpanzees (Pan trogylodytes schweinfurthii) in Western Tanzania. American Journal of Tropical Medicine and Hygiene 2010 82:967-970.

\section{doi:10.1186/1743-422X-7-303}

Cite this article as: Wevers et al:: A novel adenovirus of Western lowland gorillas (Gorilla gorilla gorilla). Virology Journal 2010 7:303.

\section{Submit your next manuscript to BioMed Central and take full advantage of:}

- Convenient online submission

- Thorough peer review

- No space constraints or color figure charges

- Immediate publication on acceptance

- Inclusion in PubMed, CAS, Scopus and Google Scholar

- Research which is freely available for redistribution

Submit your manuscript at www.biomedcentral.com/submit
C Biomed Central 